\title{
Diagnostic Pleural Fluid Cytology of Nocardial Infection in A Patient of Systemic Lupus Erythematosus
}

\author{
Sneha Singh*, Prajwala Gupta and Minakshi Bhardwaj \\ Dept of Pathology, Dr RML Hospital, New Delhi, India
}

\begin{abstract}
Systemic lupus erythematous is a chronic autoimmune inflammatory connective tissue disease. Infection remains the major cause of morbidity and mortality in such autoimmune disorders. Nocardia is an uncommon opportunistic pathogen. Due it its low incidence it is often missed by clinicians and pathologists. Delay in diagnosis and treatment is the major cause of dissemination and complications of this disease. Here we report a case of nocardiosis diagnosed on pleural fluid cytology smears using special stains.

A known case of systemic lupus erythematous diagnosed 7 years back complained of heaviness in chest, shortness of breath and fever. Ultrasonography of chest showed pleural effusion and consolidation of underlying lung. Pleural fluid was aspirated and sent for cytology. Giemsa and Papaniculaou stained smears showed dense degenerated acute and chronic inflammatory cells in a necrotic background with negatively stained shadows which were negative for $\mathrm{ZN}$ and PAS stains. However, gram stain and modified $\mathrm{ZN}$ stain $(1 \% \mathrm{H} 2 \mathrm{SO} 4)$ showed positively stained clumps of long branching filamentous organism; consistent with Nocardia spp which was later confirmed by culture studies.

This case highlights the importance of high index of suspicion for nocardia in cytology samples of pulmonary origin, especially in cases patients with immunosuppressed states. More so special stains like Gram's stain and modified ZN stain in such cases can aid in a definitive diagnosis.
\end{abstract}

\section{Keywords: Pleural Fluid, Cytology, Special stains, Nocardia}

\section{Introduction}

Nocardiosis is an opportunistic pathogen encountered rarely by pathologists ${ }^{[1]}$. It is caused by Gram positive, weakly acid-fast filamentous aerobic organisms ${ }^{[2]}$. More than 50 species of Nocardia have been identified but Nocardia Asteroides (N. Asteroides) is the most common subtype identified in humans ${ }^{[3,4]}$. People with depressed immune status are more susceptible to Nocardial infection ${ }^{[5]}$.The clinical spectrum of nocardial infection ranges from a self-limiting, subclinical condition to life-threatening disseminated disease which can be treated but needs an early diagnosis and appropriate treatment ${ }^{[6]}$. Hence, a high index of suspicion and aggressive approach to diagnosis and therapy are essential for management.

\section{Case Report}

A 37 year old female, known case of Systemic Lupus Erythematosus (SLE) with grade 4 lupus nephritis came to the hospital with chief complaints of heaviness in chest, left side chest pain for 2 days and fever with shortness of breath for 15 days. There was no history of cough with or without expectoration or significant weight loss. General examination revealed pallor and pedal oedema. Respiratory system examination showed decreased breath sounds on left side of chest. Other systems showed no significant findings. Ultrasonography of chest showed moderate left side effusion with consolidation of underlying lung. High resolution computed tomography (CT) of chest showed mild pericardial effusion, left sided pleural effusion with collapsed lung segment and atelactatic change in left upper lobe. Multiple subpleural and perilymphatic nodules in left upper lobe were also seen. Hematological laboratory work up showed haemoglobin of $7.8 \mathrm{gm} / \mathrm{dl}$, a WBC count of $15,000 / \mathrm{mm}^{3}$ with neutrophilic leucocytosis. Viral serology was non-reactive for HIV and Hepatitis B\& C. Kidney function tests were mildly deranged (urea $-77 \mathrm{mg} / \mathrm{dl}$, creatinine $1.7 \mathrm{mg} / \mathrm{dl}$, uric acid- $10.2 \mathrm{mg} / \mathrm{dl}$ ). Other routine biochemical investigations were within normal range. Pleural tap aspirated from left side yielded thick purulent material mixed with blood. Its biochemical analysis revealed sugar- $10.0 \mathrm{gm} / \mathrm{dl}$, protein-3.0gm/ dl. A presumptive clinical diagnosis of tuberculosis or fungal infection was kept. Pleural fluid sample was sent for cytological examination. Giemsa and Papaniculaou stained smears (Figure-1) showed dense degenerated acute and chronic inflammatory cells in a necrotic background with negatively stained shadows. Zeihl Nelson (ZN) and Periodic Acid Schiff's (PAS) stain were negative and did not reveal any pathogen. However, Gram stain and modified $\mathrm{ZN}$ stain $(1 \% \mathrm{H} 2 \mathrm{SO} 4)$ (Figure-2) showed positively stained clumps of long branching filamentous organism; consistent with Nocardia spp. Sample sent for 
culture confirmed presence of colonies of N. asteroides. Patient was started on combination of tablet trimethoprimsulfamethoxazole and intravenous amikacin to which the patient responded favourably.

\section{Discussion}

Nocardia is an uncommon opportunistic pathogen caused by Gram positive, weakly acid-fast filamentous aerobic organisms found worldwide ${ }^{[1]}$. Human infection occurs by direct inhalation of Nocardia species causing the commoner pulmonary manifestations or from inoculation

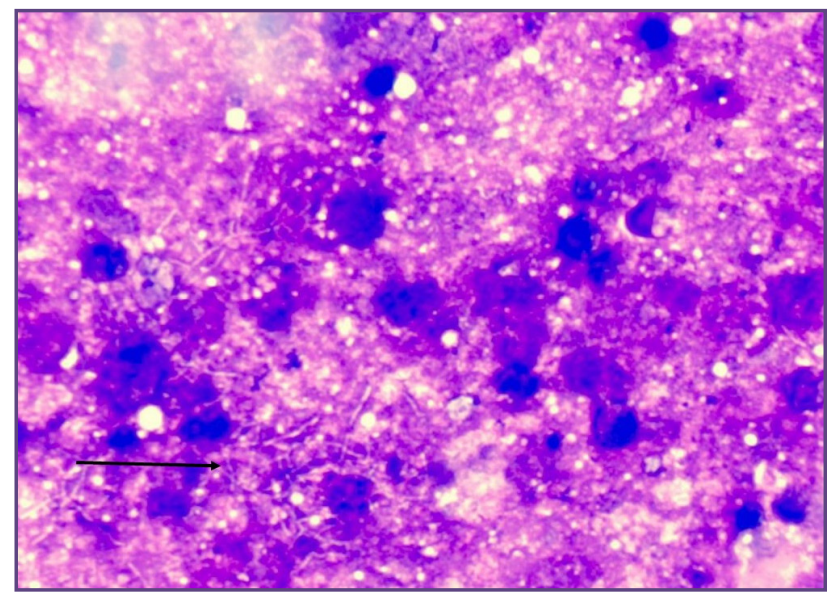

Fig. 1: Photomicrograph of pleural fluid cytology smears. MGG stain(1000x) showing and negatively stained shadows (arrow). of skin or soft tissue. In the Nocardia genus, N. asteroides is the commonest opportunistic pathogen followed by $\mathrm{N}$. brasiliensis ${ }^{[3,4]}$. Pleural involvement in nocardiosis is uncommon and is in form of pyopneumothorax, pleural effusion and empyema ${ }^{[4]}$. Patients with depressed cellular immunity are at higher risk for Nocardia infections like patients with acquired immunodeficiency syndrome, renal transplant, haematological and solid organ malignancies and long term steroid or immunotherapy ${ }^{[7]}$. SLE is a chronic autoimmune inflammatory connective tissue disease that

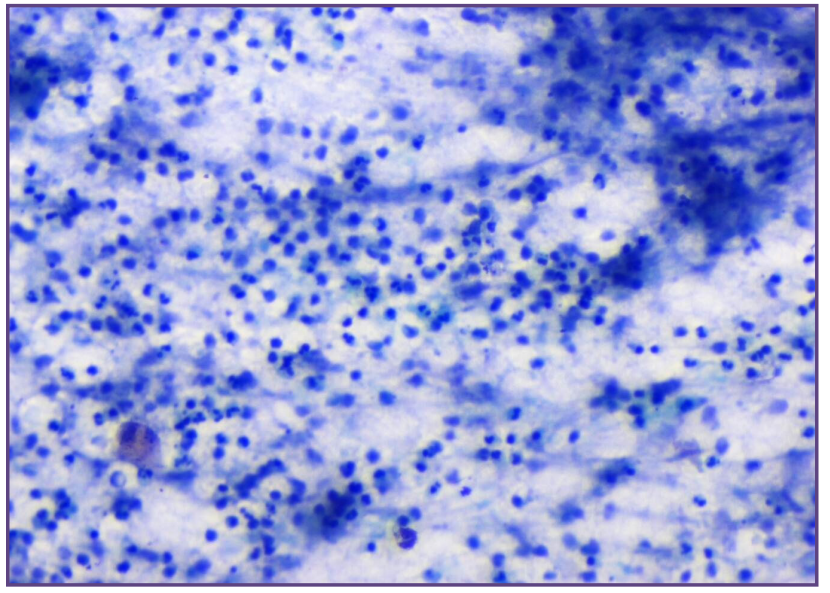

Fig 2. Photomicrograph of pleural fluid cytology smears. PAP (200x) showing degenerated inflammatory cells and necrosis.

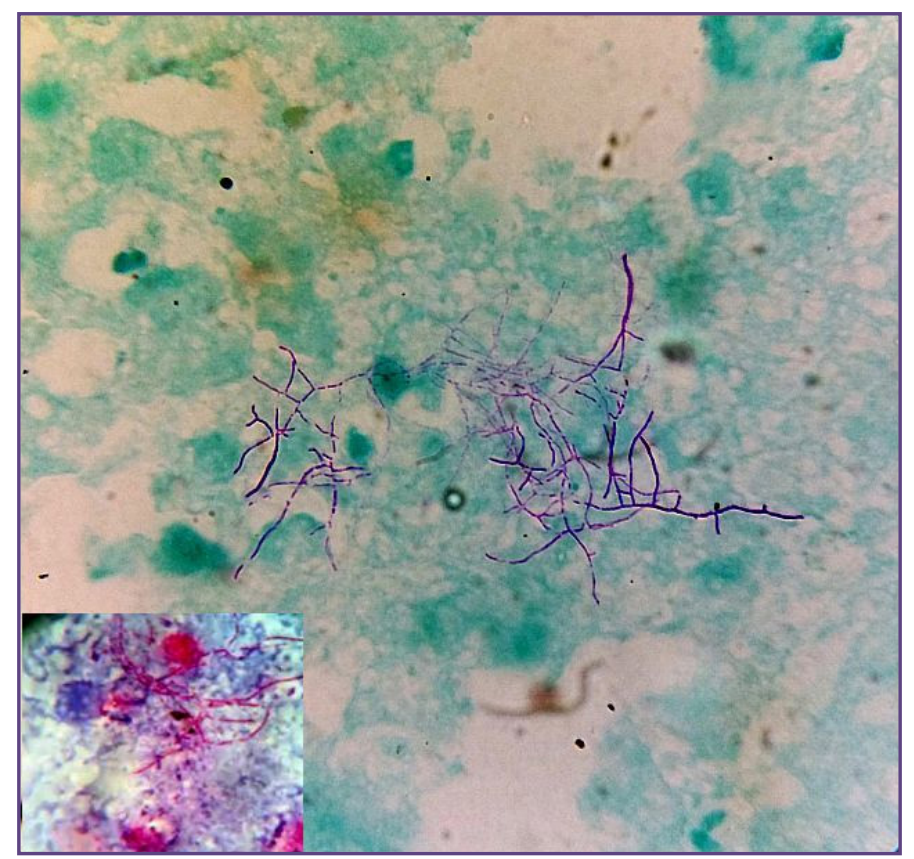

Fig. 3; Photomicrograph of special stains on pleural fluid smears. Modified ZN stain (1000x) - Showing positively stained clumps of long branching filamentous bacteria and Inset- Grams stain (1000x). 
may involve any part of body. Infection remains the major cause of morbidity and mortality in SLE patients ${ }^{[8]}$. The incidence of Nocardia infection in SLE varies between 0.6 and $2.8 \%{ }^{[9]}$. The diagnosis of pulmonary nocardial infection is often difficult due to its clinical and radiological findings overlapping and mimicking tuberculosis, fungal infection or malignancy. Demonstration of Nocardia can pose problems both in cytology and microbiology. Simultaneous positivity of culture and smears are seen in only one third cases ${ }^{[3]}$. Demonstration of Nocardia on culture media poses difficulty as negative bacterial culture specimens are usually discarded after 72 hours however nocardial culture usually needs 3-5 days to grow on routine culture media ${ }^{[3]}$. Also organism may fail to grow on culture media sometimes due to low pathogen amount in the sample ${ }^{[7]}$. Background of dense suppurative inflammation, necrosis and light staining on routine cytological stains causes difficulty in identifying nocardia on cytology ${ }^{[10]}$. There have been few case reports demonstrating nocardia on cytological examination of pleural fluid ${ }^{[5]}$.

\section{Conclusion}

This case highlights the importance of high index of suspicion for this rarely encountered organism in cytology smears, especially in cases with immunosuppressed states. More so use of special stains like Gram's stain and modified ZN stain in such cases can aid in a definitive diagnosis.

\section{Reference}

1. Hu Y., Wang L., Huang X., Liang Y. and Zhang J. Systemic Nocardia brasiliensis infection in a patient with systemic lupus erythematosus: successful diagnosis and therapy. Int J Dermatol, 2016; 55: 453-9.

2. Li S., Song X. yu, Zhao Y. yue, Xu K., Bi Y., Huang H., $\& \mathrm{Xu}$ Z. jun. Clinical Analysis of Pulmonary Nocardiosis in Patients With Autoimmune Disease. Medicine,2015;94, e1561.

3. Gowrinath K, Rao PS, Mohapatra AK, Prakash PY. Pleural nocardiosis. Indian J Chest Dis Allied Sci. 2009;51:169-71.

4. Bagali, S., \& Mantur, P. Pleural Nocardiosis in an Immunocompetent Patient: A Case Report. Journal of Clinical and Diagnostic Research: JCDR, 2016;10, DD01-2.

5. R. Kumar, D. Chhina, V. Kaushal, R. Mahajan, and H. Kaur, Cytological diagnosis of pulmonary nocardiosis in an immunocompromised patient. Indian Journal of Medical Microbiology. 2008; 26: 380-2.

6. Singh A, Chhina D, Soni RK, Kakkar C, Sidhu US. Clinical spectrum and outcome of pulmonary nocardiosis: 5-year experience. Lung India. 2016;33:398-403.

7. Hui CH, Au VWK, Rowland K, Slavotinek JP, Gordon DL: Pulmonary nocardiosis re-visited: Experience of 35 patients at diagnosis. Respir Med. 2003;97:709-717.

8. Cojocaru M, Cojocaru IM, Silosi I, Vrabie CD. Manifestations of Systemic Lupus Erythematosus. Maedica. 2011;6:330-6.

9. Leong KP, Tee NW, Yap WM, Chee TS, Koh ET. Nocardiosis in patients with Systemic lupus erythematosus. The Singapore Lupus Study Group. J Rheumatol. 2000;27:1306-12.

10. Mathur S, Sood R, Aron M, Iyer VK, Verma K. Cytologic diagnosis of pulmonary nocardiosis: A report of 3 cases. Acta Cytol. 2005;49: 567-570. 